\title{
Phytochemical Composition of Solanum retroflexum Analysed with the Aid of Ultra-Performance Liquid Chromatography Hyphenated to Quadrupole-Time-of-Flight Mass Spectrometry (UPLC-qTOF-MS)
}

\author{
Grace Daji, ${ }^{1}$ Paul Steenkamp $\mathbb{D}^{2},{ }^{2}$ Ntakadzeni Madala $\mathbb{D}^{2},{ }^{2}$ and Bhekisisa Dlamini $\mathbb{D}^{1}$ \\ ${ }^{1}$ Department of Biotechnology and Food Technology, Faculty of Science, University of Johannesburg, P.O. Box 17011, \\ Doornfontein Campus, Gauteng 2028, South Africa \\ ${ }^{2}$ Department of Biochemistry, University of Johannesburg, P.O. Box 524, Auckland Park 2006, South Africa
}

Correspondence should be addressed to Bhekisisa Dlamini; bcdlamini@uj.ac.za

Received 28 May 2018; Revised 28 July 2018; Accepted 13 August 2018; Published 18 September 2018

Academic Editor: Luca Campone

Copyright (c) 2018 Grace Daji et al. This is an open access article distributed under the Creative Commons Attribution License, which permits unrestricted use, distribution, and reproduction in any medium, provided the original work is properly cited.

Solanum retroflexum (nightshade) is an edible plant that is consumed in some regions of South Africa. Its leaves are a good source of vitamins, proteins, and minerals. It appears that there is no scientific report about the phytochemical composition of $S$. retroflexum. Here, ultra-performance liquid chromatography coupled to quadruple-time-of-flight mass spectrometry (UPLCqTOF-MS) technique was use to achieve an untargeted metabolite fingerprinting of this plant. A total of 30 phytochemicals, including alkaloids, flavonoids, and cinnamic acids derivatives, were identified from the methanolic leaf extracts. The concentration of solvent did not affect the type of compounds extracted. The extraction resulted in qualitative yields of molecules such as quercetin-3-rutinoside, kaempferol-3-0-rutinoside, kaempferol-3-0-glucoside, 3-caffeoylquinic acid, 5-caffeoylquinic acid, and 3, 4-di-caffeoylquinic acid. The present study confirms the presence of phytochemical compounds in S. retroflexum similar to other Solanum plants.

\section{Introduction}

Solanum plants (family Solanaceae) have been widely explored for their phytochemical constituents. These bioactive compounds have been isolated from various parts such as the leaves, fruits, and roots [1-3]. The presence of various phytochemicals in Solanum plants has allowed for their use in medicine and food as functional foods or dietary supplements [4]. The type of phytochemical compounds found in Solanum plants vary with species, plant part, and the extraction method [5]. The presence of tannins, flavonoids, alkaloids, glycosides, steroids, and terpenoids has been reported in S. nigrum L. leaves, fruits, and stem [3]. Phenolic compounds such as chlorogenic acid, caffeic acid, rosmarinic acid, gallic acid, and flavonoids have been identified together with alkaloids in S. corymbiflorum leaves [2]. The presence of some compounds such as alkaloids in food can cause toxicity to humans depending on dosage. At low doses, the intake of glycoalkaloids may cause gastrointestinal disturbance such as vomiting, diarrhea, and abdominal pain, while at high doses, it may lead to serious complications such as rapid pulse and in some cases, coma and death [6].

Extraction of phytochemicals from plants is generally done with conventional extraction methods such as the use of organic solvents. However, there are concerns about the potential health threatening effects of organic solvents. Despite this, the use of organic solvents such as methanol is known to produce good extraction yields. In addition, aqueous methanol has been reported to extract higher amounts of phenolic compounds compared to absolute methanol. Quadruple time-of-flight mass spectrometry (qTOF-MS) fingerprinting method is one of the improved 
methods that has been proved to generate stable and reproducible results [7]. Coupling the HPLC with MS does not only offer excellent sensitivity and selectivity but also allows for determination of unknown and known phytochemical compounds [8]. The qTOF-MS fingerprinting method has been proved to generate stable and reproducible results, particularly in the identification of chlorogenic acid [9]. It appears that there is no scientific report on the phytochemistry of Solanum retroflexum despite the fact that the people in Venda region, Limpopo province, South Africa, consume the leaves as food. Therefore, the aim of this research was to investigate the phytochemical composition of S. retroflexum leaf extracts with the aid of UPLC-qTOF-MS.

\section{Materials and Methods}

2.1. Plant Collection. Solanum retroflexum leaves were collected from the Venda region, Limpopo province, South Africa, between September and October 2015. The leaves were air-dried under shade and stored in airtight containers at room temperature for further use.

2.2. Extraction of Metabolites. Three different types of sample extracts were obtained by using aqueous methanol (40\%, 60\%, and $80 \%$, methanol/ water, v/v) solution as solvent. Solanum retroflexum leaf samples were extracted following the method described by Abu-Reidah et al. [10] and Ramabulana et al. [11], with slight changes. The grounded $(2.0 \mathrm{~g})$ leaves of $S$. retroflexum were extracted with different concentrations of aqueous methanol and sonicated at room temperature for 15 minutes. The resultant mixture was centrifuged for 10 minutes at $5000 \mathrm{~g}$ and evaporated using a Buchi rotary evaporator to approximately $1 \mathrm{~mL}$. The extracts were then dried to completeness overnight, dissolved again in methanol, and filtered through $0.22 \mu \mathrm{m}$ syringe filters and kept at $-20^{\circ} \mathrm{C}$ prior to analysis.

\subsection{Ultrahigh-Performance Liquid Chromatography and} Mass Spectrometry. Chromatographic separation was conducted according to the procedure described by Ramabulana et al. [11] using an Acquity UHPLC connected to a Synapt G1 qTOF-MS detector (Waters Corporation, MA, USA). The mobile phase consisted of formic acid $(0.1 \%)$ in deionised water (solvent A) and acetonitrile with $0.1 \%$ formic acid (Romil Pure Chemistry, Cambridge, UK) (solvent B). Chromatographic separation was achieved using a 30 min gradient elution method consisting of the following settings: the initial conditions were $98 \% \mathrm{~A}$ at a flow rate of $0.4 \mathrm{~mL} / \mathrm{min}$. The initial conditions were kept constant for $1 \mathrm{~min}$. Conditions were changed to $97 \% \mathrm{~A}$ at $3 \mathrm{~min}$, reduced slightly to $92 \%$ A at $4 \mathrm{~min}$, held for $21 \mathrm{~min}$, and then changed to $50 \% \mathrm{~A}$ at $25 \mathrm{~min}$. Conditions were changed to $5 \% \mathrm{~A}$ at $26 \mathrm{~min}$ and kept for $2 \mathrm{~min}$ and finally returned to initial conditions of $98 \% \mathrm{~A}$ at $28 \mathrm{~min}$ and kept constant for another 2 min to allow reequilibration before the next run. Elution was monitored using a photodiode-array detector (PDA) collecting 20 spectra per second between the 200 and $500 \mathrm{~nm}$ range. For mass spectrometry, the acquisition parameters discussed by Ramabulana et al. [11] were followed. Briefly, MS data were acquired using both positive and negative ESI modes. The MS was configured to scan the range of 100$1000 \mathrm{Da}$ with a scan time of $0.2 \mathrm{~s}$. After series of optimization, the following settings were found to be optimal: capillary voltage of $2.5 \mathrm{kV}$, sample cone potential of $30 \mathrm{~V}$, source temperature of $120^{\circ} \mathrm{C}$, desolvation temperature of $450^{\circ} \mathrm{C}$, cone gas flow of $50 \mathrm{~L} / \mathrm{h}$ and desolvation gas flow of $550 \mathrm{~L} / \mathrm{h}$, and multichannel plate detector potential of $1600 \mathrm{~V}$. In order to achieve efficient fragmentation to aid during identification, data were collected using collision energy ramp of $10-30 \mathrm{eV}$ and, when necessary, a higher collision energy ramp of $165-60 \mathrm{eV}$ was also used. Unless stated, otherwise only ESI negative data are reported herein.

\section{Results and Discussion}

3.1. Methanol Extraction of Phytochemicals. Different concentrations of methanol $(40 \%, 60 \%$, and $80 \%$, methanol/water, $\mathrm{v} / \mathrm{v}$ ) were used in this study to understand different degrees of the extractive potency of different concentrations of the solvent. Screening of metabolites was achieved by an untargeted UPLC-qTOF-MS at positive and negative ionization modes. For efficient metabolite identification, MS fragmentation patterns were used in conjugation with standards. The methanol extracts of Solanum retroflexum generated using the different concentrations of aqueous methanol resulted in very similar phytochemical composition (Figure 1). A total of 30 metabolites were identified, and these include chlorogenic acids (CGAs), flavonoids, and alkaloids as shown in Table 1.

3.2. Chlorogenic Acids. Chlorogenic acids are phenolic acids that result from the esterification of quinic acid (QA) and cinnamic acid (CA) derivatives including caffeic, sinapic, and ferulic acid [12]. In this study, chlorogenic acids were identified chromatographically with the aid of our recently developed in-source collision induced dissociation method. Generally, CGAs result in very similar fragments characterised by the presence of Q1 [quinic acid-H], C1 [caffeic acid-H], Q2 [quinic acid- $\mathrm{H}_{2} \mathrm{O}$ ] and $\mathrm{C} 2$ [caffeic acid- $\mathrm{CO}_{2}$ ] fragment ions [13]. Four peaks at $m / z 353$ with region isomers and geometric isomers of caffeoylquinic acid (CQAs) were detected in the extracts of S. retroflexum leaves (Figure 2). The CQA derivatives, the most common form of CGA, are formed from the esterification of quinic acid to one or more caffeic acid unit. The metabolites from CGAs and their isomers (positional) can be differentiated mainly by fragmentation patterns into 3 CQA, 4 CQA, and 5 CQA [14].

Chromatographically, it is easy to identify CGA and also differentiate between the trans and cis isomers because of the order in which they elute [9]. It is worth noting that these isomers (trans and cis) are known to have similar fragmentation patterns [15]. Here, both the trans and cis isomers were identified (Table 1). This can be attributed to too much exposure of the leaves to UV rays since the S. retroflexum came from the northern part of South Africa that is known to be very hot. Ultraviolet rays are known to convert trans to cis isomer [16]. The presence of these cis isomers is important 


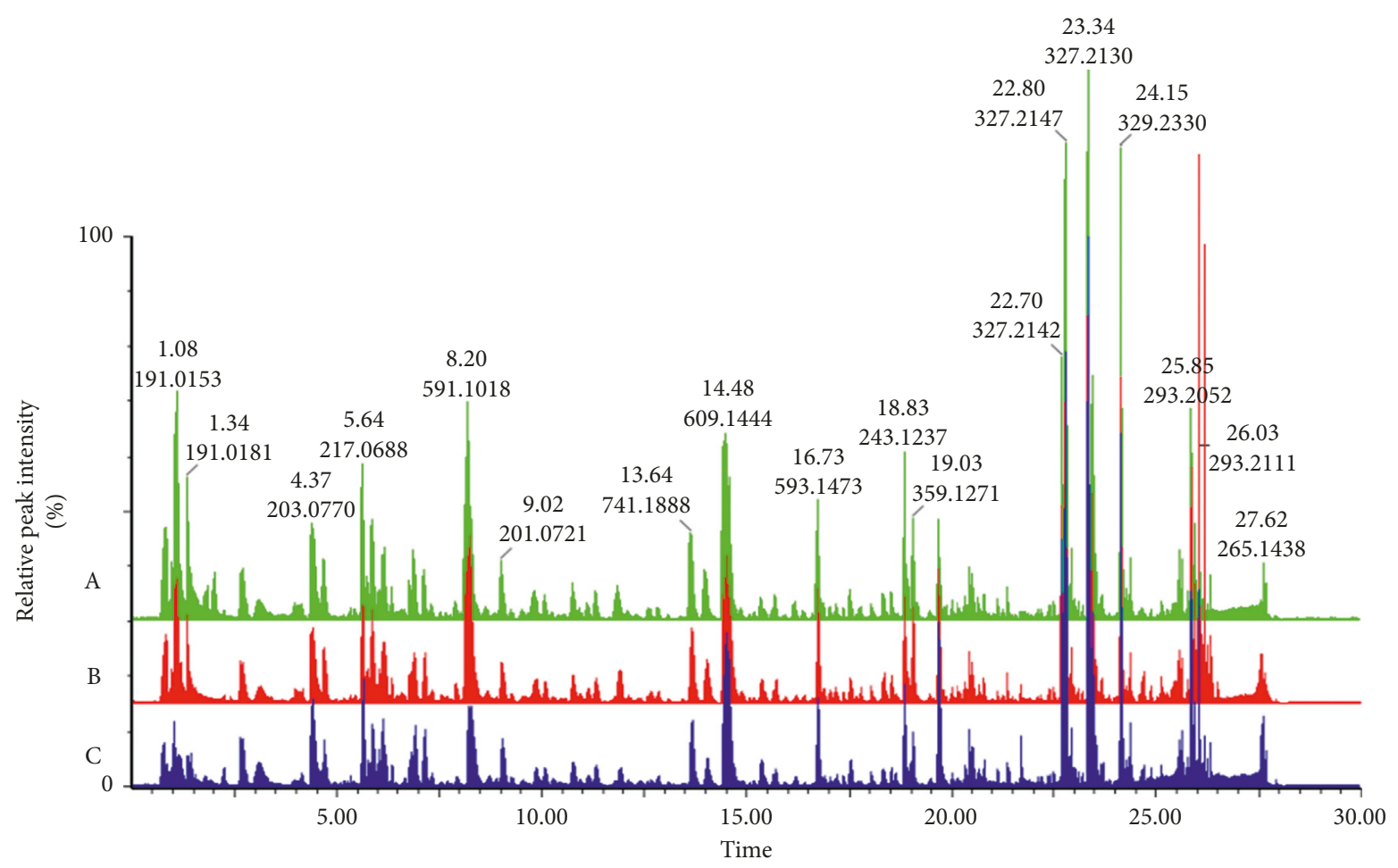

FIGURE 1: UHPLC-qTOF-MS chromatogram of 40\% (a), 60\% (b), and 80\% (c) aqueous methanol Solanum retroflexum leaf extracts showing peaks detected in the negative- and positive-ionization mode.

since they are known to possess biological activities such as antioxidant activity, antimicrobial activity, antiviral activity, and antidiabetic activity [17].

The presence of CGAs in Solanum leaves has previously been reported in the Solanaceae family but not in $S$. retroflexum. For instance, CGAs were identified in Lycium intricatum methanolic leaves extract [10]. Solanum corymbiflorum leaves extracted with ethanol were also found to contain high amounts of CGAs [2]. Previous research has shown that CGAs may exhibit antibacterial, antiviral, and antioxidant activities [18]. In addition, anti-inflammatory activities of CGAs possibly by modulating a number of important metabolic pathways have been reported elsewhere [19]. Chlorogenic acids are also found in beverages such as tea, coffee, and wine. They could be responsible, in part, for the health promoting benefits of such food products.

Apart from the CQAs, other monoacyl CGA that are not ferulic but coumaric and ferulic were identified in this study. Also, diacyl CGAs which are very important molecules with good biological activity, such as being natural antioxidants in food or nonfood products, were observed.

3.3. Characterisation of Caffeoylquinic Acids. Molecules 1, 2, and 3 were identified as 3CQA, 4CQA, and 5CQA, respectively (Table 1 ). Molecule $\mathbf{1}$ at retention time $9.8 \mathrm{~min}$ produced a precursor ion at $m / z 353$ identified as cis-3CQA. This molecule produced fragments ions at $\mathrm{m} / z 179,191$, and 135 characteristic of quinic acylated at 3 positions with caffeoylquinic acids, thus 3CQA [20]. Molecules 2 and 3 were identified as trans-4CQA and 5CQA with a precursor ion at 191, 179, 173, and 135. Molecules 4 and 5 were identified as cis-4CQA and 5CQA. The CQAs are the most researched amongst the CGAs because they widely occur in nature [9]. It was also noted that cis-5-CQA showed a high intensity than cis-3-CQA (Figure 2). It has also been reported that the same molecule is induced by activators responsible for plant defence response [21].

3.4. Characterisation of Feruloylglycoside. Feruloylglycosides have a molecular weight (Mr) of 356, and they produce a precursor ion at $m / z 355$. Here, only one molecule was identified, and it had a base peak at $m / z 175$ and 160 ([ferulic acid-H- $\left.\mathrm{H}_{2} \mathrm{O}\right]$ ) (Table 1). The fact that feruloylglycoside molecule is biosynthesised in Solanum retroflexum leaves suggests a remarkable biochemical feature of this plant.

3.5. Characterisation of Di-caffeoylquinic Acid and Caffeoylquinic Acid-glucoside. Both di-caffeoylquinic acid and caffeoylquinic acid glucoside were identified with a precursor ion at $m / z 515$ (Table 1). These molecules can be identified separately using their accurate masses and fragmentation patterns [22]. Here, molecules 7-13 were identified as either di-CQA or CQA-glycoside. Previous research indicate that $d i$-CQA and CQA glycosides have an average $\mathrm{m} / z$ of $515.1463\left(\mathrm{C}_{25} \mathrm{H}_{23} \mathrm{O}_{12}\right)$ and $515.1292\left(\mathrm{C}_{22} \mathrm{H}_{27} \mathrm{O}_{14}\right)$, respectively, based on accurate mass [22]. Molecules 7 and 8 were identified as trans- and cis-CQA-glycoside. The CQA glycoside produced unique ions that did not occur in the 
TABLE 1: Compounds identified with UHPLC in Solanum retroflexum leaf aqueous methanol extracts.

\begin{tabular}{|c|c|c|c|c|}
\hline Mol. no. & Retention time (min) & Mass & Compound name & Diagnostic $m / z$ ions \\
\hline 1 & 9.8 & 353 & 3-CQA & $191,179,135$ \\
\hline 2 & 5.6 & 353 & trans-4-CQA & $191,135,173,179$ \\
\hline 3 & 5.1 & 353 & trans-5-CQA & 191,135 \\
\hline 4 & 6.0 & 353 & cis-4-CQA & $191,135,173,179$ \\
\hline 5 & 5.9 & 353 & cis-5-CQA & 191,135 \\
\hline 6 & 6.5 & 355 & Feruroylglycoside & 160,175 \\
\hline 7 & 5.3 & 515 & trans-5-CQA glycoside & 191,135 \\
\hline 8 & 6.4 & 515 & cis-5-CQA glycoside & 191,135 \\
\hline 9 & 8.9 & 515 & 3, 4-di CQA & $191,179,173,135$ \\
\hline 10 & 9.1 & 515 & $c i s-3,5-d i-C Q A$ & $191,179,135$ \\
\hline 11 & 9.3 & 515 & trans-3, 5-di-CQA & $191,179,135$ \\
\hline 12 & 9.5 & 515 & trans-4, 5-di-CQA & $191,179,135$ \\
\hline 13 & 10.5 & 515 & $c i s-4,5-d i-\mathrm{CQA}$ & $191,179,135$ \\
\hline 14 & 5.3 & 337 & 3-p-CoQA & 177 \\
\hline 15 & 6.8 & 337 & trans-5-p-CoQA & 191 \\
\hline 16 & 7.5 & 337 & $c i s-5-p-\mathrm{CoQA}$ & 191 \\
\hline 17 & 8.7 & 339 & Coumaroyl-hexose & $223,164,149$ \\
\hline 18 & 7.4 & 337 & 4-p-CoQA & 173 \\
\hline 19 & 7.4 & 367 & trans-5-FQA & 191 \\
\hline 20 & 8.0 & 367 & cis-5-FQA & 191 \\
\hline 21 & 10.7 & 367 & 4-FQA & 173 \\
\hline 22 & 10.3 & 367 & 3-FQA & 193,134 \\
\hline 23 & 8.1 & 609 & Quercetin-3-rutinoside & 300 \\
\hline 24 & 9.1 & 447 & Kaempferol-3-glucoside & 284 \\
\hline 25 & 8.4 & 463 & Quercetin-7-glucoside & 300 \\
\hline 26 & 8.8 & 593 & Kaempferol-3-0-rutinoside & 285 \\
\hline 27 & 9.0 & 623 & Isorhamnetin-3-0-rutinoside & 315 \\
\hline 28 & 8.7 & 447 & Quercetin-3-rhamnoside & 300 \\
\hline 29 & 20.3 & 884 & Solasonine & $722,576,414$ \\
\hline 30 & 20.1 & 868 & Solamargine & $722,576,414$ \\
\hline
\end{tabular}

Note. CQA, caffeoylquinic acid; di-CQA, di-caffeoylquinic acid; $p$-CoQA, $p$-coumaroylquinic acid; FQA, feruloyquinic acids; mol no., molecule number; min, minutes; $m / z$, mass-to-charge ratio; DNP, dictionary of natural products 2015 [37]; KMI, KNApSAcK Metabolites Information 2015 [38].

di-CQA MS spectra. These were at $\mathrm{m} / z 341$ ([caffeoyl glucoside-H]-) and/or at $\mathrm{m} / z 323$ (caffeoyl glucoside-H$\left.\mathrm{H}_{2} \mathrm{O}\right]-$ ). Recently, the hierarchical fragmentation schemes of similar molecules have been reported [23]. Their work noted the formation of a glycoside by CQA through an ether bond at either C-3 or C-4 on the aromatic caffeoyl ring. Molecules 9-13 were identified as di-CQAs (3, 4 di-CQA (9), trans- and cis-3, $5 d i$-CQA (10 and 11), and trans- and cis-4, $5 d i-\mathrm{CQA}$ (12 and 13).

3.6. Characterisation of p-Coumaroylquinic Acid and Coumaroyl-hexose. The $S$. retroflexum leaf extracts showed precursor ions at $\mathrm{m} / \mathrm{z} 337$, and these were identified as p-coumaroylquinic acid ( $\mathrm{Mr}$ of 338) (Table 1). These ion peaks were identified as 3-pCoQA (14), trans $5-p \mathrm{C}_{\mathrm{O}} \mathrm{QA}(\mathbf{1 5})$, cis-5-p $\mathrm{C}_{\mathrm{O}} \mathrm{QA}(\mathbf{1 6})$, and $4-p-\mathrm{C}_{\mathrm{O}} \mathrm{QA}(\mathbf{1 8})$. The presence of $p$ CoQA is not surprising since it has also been identified in leguminous plants and birch trees [24]. Molecule 17 was identified as Coumaroyl-hexose with a precursor ion at $\mathrm{m} / \mathrm{z}$ 339 and product ions at 223, 164 and 149.

3.7. Characterisation of Feruloylquinic Acids. The identification of feruloylquinic acids is based on that fact that they have a Mr of 368. In this study, molecules harbouring ferulic acid moieties were observed. This was similar to what occurred with $p$ CoQA and CQA. Here, the peaks identified as feruloylquinic acids were trans-5-FQA (19), cis-5-FQA (20), 4-FQA (21), and 3-FQA (22) (Table 1).

3.8. Characterisation of Flavonoid Derivatives. A total of six flavonoids 23, 24, 25, 26, 27, and 28 were detected and characterised in S. retroflexum by QTOF-MS method presented herein (Table 1). Molecule 23 at retention time $8.12 \mathrm{~min}$ produced a precursor ion at $\mathrm{m} / \mathrm{z} 609$, and its fragmentation pattern was found consistent with that of quercetin-rutinoside in the literature [25]. Briefly, the product ions of molecule $\mathbf{2 3}$ were primarily caused by the exclusion of a rutinoside sugar moiety, which resulted in an intense peak at $m / z$ 300. Importantly, quercetin is reported to be a crucial dietary flavonoid that possesses health benefits such as suppressing chronic diseases [26]. This has made it useful for the treatment of certain chronic diseases. Furthermore, quercetin has other biological potential associated with protection of liver cells and prevention of suppressing oxidation of the haemoglobin as a result of its antioxidant activity [27].

Molecule 24 had a precursor ion at $m / z 447[\mathrm{M}-\mathrm{H}]^{-}$, and it was identified as kaemferol-3-glucoside (Table 1). It produced fragment ions at $\mathrm{m} / \mathrm{z} 284$ which is thought to be 




(a)

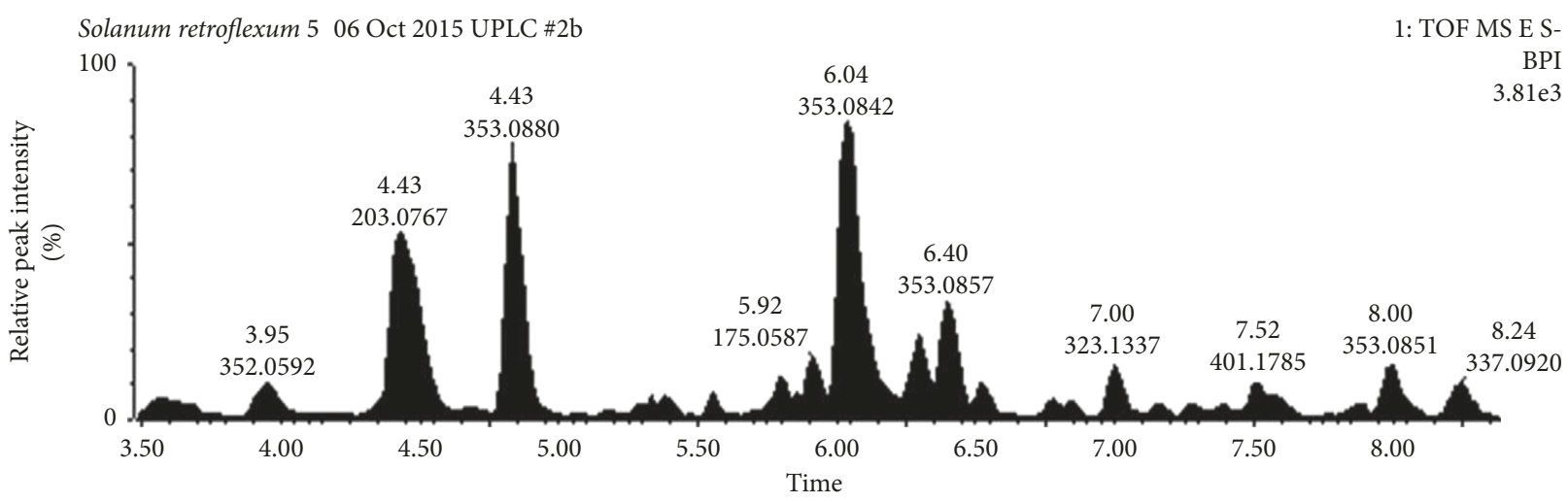

(b)

FIGURE 2: UHPLC-qTOF-MS chromatogram showing single-ion chromatogram (a) and the base peak in chromatogram (b) of chlorogenic acid detected in the negative-ionization mode.

due to the loss of a hexose moiety [28]. Molecule 25 was identified as quercetin-7-glucoside because it had a precursor ion at $m / z 463[\mathrm{M}-\mathrm{H}]^{-}$and a fragment ion produced at $m / z$ 300. Molecule 26 was identified as kaempferol-30 rutinoside with a precursor ion at $m / z 593[\mathrm{M}-\mathrm{H}]^{-}$and a fragment ion at $m / z$ 285, indicating the elimination of a rutinoside moiety. Such a fragment pattern has been reported elsewhere [29]. Molecule 27 had a precursor and fragment ions at $m / z 623[\mathrm{M}-\mathrm{H}]^{-}$and $m / z 315$, respectively. It was identified as isorhamnetin-3-0-rutinoside. According to Makita et al. 2016 [25], the absence of a rutinoside moiety makes it to represent an isorhamnetin aglycone. Molecule $\mathbf{2 8}$ was identified as quercetin-3-rhamnoside because of the presence of a precursor ion at $\mathrm{m} / z 447[\mathrm{M}-\mathrm{H}]^{-}$and the fact that it produced a fragment ion at $\mathrm{m} / z 300$.

In foods, flavonoids occur as 0-glycosides with sugar bound at $\mathrm{C}_{3}$ position. The structures of quercetin-3rutinoside, kaempferol-3-glucoside, quercetin-7-glucoside, kaempferol-3-0-rutinoside, isorhamnetin-3-0-rutinoside, and quercetin-3-rhamnoside identified flavonoid glycosides show that almost all of them contain either kaemferol, quercetin, or isorhamnetin core moieties. These indicate that the flavonoid core structures have sugar moieties attached to them. The sugar moiety is important in the bioavailability of flavonoid glycosides in humans. The total number of sugar moieties, their position, and structure influences the antioxidant activity of such flavonoids [30].

Polyphenol metabolites such as flavonoids have been reported to possess various medicinal and pharmacological activities [31]. These properties are thought to be affected by the configuration structure of the flavonoid molecule. Therefore, it is not surprising that flavonoids such as quercetin have been utilised in clinical trials. High levels of quercetin and kaemferol in Solanum lycopersicum have been reported to exhibit antioxidant and pharmacological properties [32]. Flavonoids isolated from Solanum torvum have also been shown to have important antimicrobial activity. Flavonoids are thought to inhibit microbial growth through formation of complexes with bacterial cell walls [33]. Kaemferol, quercetin, and isorhamnetin aglycone are the most common flavonoids that are abundant in plant tissues [25].

3.9. Characterisation of Alkaloid Derivatives. The S. retroflexum methanol leaf extracts were found to contain two alkaloids. Molecules $\mathbf{2 9}$ and $\mathbf{3 0}$ with precursor ions at $\mathrm{m} / \mathrm{z}$ 884 and 868 were identified as solasonine and solamargine, respectively (Table 1 ). The mass spectra of these molecules are shown in Figure 3. The difference in the $m / z$ ratios of the 




(a)



(b)

FIgURE 3: Mass spectra showing fragmentation pattern of solasonine (a) and solamargine (b).

identified Alkaloids is due to their aglycone variations. Despite this, their equivalent glycosides constitute either a solatriose or chacotriose as a sugar moiety. Thus, solasonine has a molecular ion at $m / z 884$, and solamargine has one at $m / z$ 868. In addition, the fragment ions at $m / z 722,576$, and 141 were identical, and they elute very close to each other. 
Glycoalkaloids (GAs) function chemically as defence compounds against herbivores, microorganisms, and competing plants. As a survival strategy, plants need to store sufficient amount of these defence compounds at strategic sites such as the bark, leaves, flower, seeds, or fruits [34]. Therefore, the presence of steroidal glycoalkaloid in $S$. retroflexum leaves should not be neglected because of its potential toxicity to humans. Solasonine and solamargine have also been identified in the leaves of Solanum incanum [35]. In their study, it was reported that the concentration of these alkaloids declined during the plants developmental stages with the least levels recorded at maturity. Other Solanum plants such as Solanum nigrum have been reported to produce solasonine and solamargine [36]. At low doses, the intake of glycoalkaloids may cause gastrointestinal disturbances such as vomiting, diarrhea, and abdominal pain, while at high doses, it may lead to serious complications such as rapid pulse and in some cases, coma and death [6].

\section{Conclusions}

According to our knowledge, this is the first comprehensive study on the phytochemical composition of Solanum retroflexum using the UHPLC-qTOF-MS method. A total of 30 different metabolites were identified with methanol extraction, and these include alkaloids, flavonoids, and cinnamic acid derivatives. The concentration of solvent did not affect the type of compounds extracted. The present study confirms the presence of phytochemical compounds in $S$. retroflexum similar to other Solanum plants.

\section{Data Availability}

The data used to support the findings of this study are included within the article.

\section{Conflicts of Interest}

All authors declare that they have no conflicts of interest.

\section{Acknowledgments}

The authors thank the South African National Research Foundation (NRF) and University of Johannesburg for financial support.

\section{References}

[1] Z. Yousaf, Y. Wang, and E. Baydoun, "Phytochemistry and pharmacological studies on Solanum torvum," Journal of Applied Pharmaceutical Science, vol. 3, no. 4, pp. 152-160, 2013.

[2] M. Piana, C. Camponogara, and A. A. Boligon, "Topical antiinflammatory activity of Solanum corymbiflorum leaves," Journal of Ethnopharmacology, vol. 179, pp. 16-21, 2016.

[3] P. Kumar, J. Kumar, R. Kumar et al., "Studies on phytochemical constituents and antimicrobial activities of leaves, fruits and stems of Solanum nigrum L," Asian Journal of Plant Science and Research, vol. 6, no. 4, pp. 57-68, 2016.

[4] M. Neacsu, N. Vaughan, V. Raikos et al., "Phytochemical profile of commercially available food plant powders: their potential role in healthier food reformulations," Food Chemistry, vol. 179, pp. 159-169, 2015.

[5] B. S. Paulsen, "Bioactive compounds in plants-benefits and risks for man and animals," The Norwegian Academy of Science and Letters, vol. 50, pp. 18-29, 2010.

[6] J. Ruprich, I. Rehurkova, P. E. Boon et al., "Probabilistic modelling of exposure doses and implications for health risk characterization: Glycoalkaloids from potatoes," Food and Chemical Toxicology, vol. 47, pp. 2899-2905, 2009.

[7] P. G. Matshediso, E. Cukrowska, and L. Chimuka, "Development of pressurised hot water extraction (PHWE) for essential compounds from Moringa oleifera leaf extracts," Food Chemistry, vol. 172, pp. 423-427, 2015.

[8] J. Zhao, L. Y. Ge, W. Xiong, F. Leong, L. Q. Huang, and S. P. Li, "Advanced development in phytochemicals analysis of medicine and food dual purposes plants used in China (2011-2014)," Journal of Chromatography A, vol. 1428, pp. 39-54, 2016.

[9] E. N. Ncube, L. A. Piater, P. A. Steenkamp et al., "Analyses of chlorogenic acids and related cinnamic acid derivatives from Nicotiana tabacum tissues with the aid of UPLC-QTOF-MS/ MS based on the in-source collision-induced dissociation method," Chemistry Central Journal, vol. 8, no. 1, pp. 1-10, 2014.

[10] I. M. Abu-Reidah, M. S. Ali-Shtayeh, R. M. Jamous et al., "Comprehensive metabolite profiling of Arum palaestinum (Araceae) leaves by using liquid chromatography-tandem mass spectrometry," Food Research International, vol. 70, pp. 74-86, 2015.

[11] T. Ramabulana, R. D. Mavunda, P. A. Steenkamp et al., "Perturbation of pharmacologically relevant polyphenolic compounds in Moringa oleifera against photo-oxidative damages imposed by gamma radiation," Journal of Photochemistry and Photobiology B: Biology, vol. 156, pp. 79-86, 2016.

[12] B. S. Khoza, S. Gbashi, P. A. Steenkamp, P. B. Njobeh, and N. E. Madala, "Identification of hydroxylcinnamoyl tartaric acid esters in Bidens pilosa by UPLC-tandem mass spectrometry," South African Journal of Botany, vol. 103, pp. 95-100, 2016.

[13] Y. Che, Z. Wang, Z. Zhu et al., "Simultaneous qualitation and quantitation of chlorogenic acids in kuding tea using ultrahigh-performance liquid chromatography-diode array detection coupled with linear ion trap-orbitrap mass spectrometer," Molecules, vol. 21, no. 12, pp. 1-14, 2016.

[14] M. N. Clifford, "LC-MSn analysis of the cis isomers of chlorogenic acids," Food Chemistry, vol. 106, no. 1, pp. 379385,2008

[15] Y. Feng, C. Sun, Y. Yuan et al., "Enhanced oral bioavailability and in-vivo antioxidant activity of chlorogenic acid via liposomal formulation," International Journal of Pharmaceutics, vol. 501, no. 1-2, pp. 342-349, 2016.

[16] A. I. Romero-pe, R. M. Lamuela-ravento, A. L. Waterhouse et al., "Levels of cis- and trans-resveratrol and their glucosides in white and rose Vitis vinifera wines from Spain," Journal of Agriculture and Food Chemistry, vol. 44, pp. 2124-2128, 1996.

[17] J. L. Willems, M. M. Khamis, W. M. Saeid et al., "Analysis of a series of chlorogenic acid isomers using ion mobility tandem mass spectrometry," Analytica Chimica Acta, vol. 933, pp. 164-174, 2016.

[18] R. Upadhyay and L. J. M. Rao, "An outlook on chlorogenic acids occurrence, chemistry, technology and biological activities," Critical Reviews in Food Science and Nutrition, vol. 53, no. 9, pp. 968-984, 2013. 
[19] N. Liang and D. D. Kitts, "Role of chlorogenic acids in controlling oxidative and inflammatory stress conditions," Nutrients, vol. 8, no. 1, p. 16, 2015.

[20] J. Carlotto, L. M. da Silva, N. Dartora et al., "Identification of a dicaffeoylquinic acid isomer from Arctium lappa with a potent anti-ulcer activity," Talanta, vol. 135, pp. 50-57, 2015.

[21] A. Farah, M. Monteiro, and C. M. Donangelo, "5-Ocaffeoylquinic acid (5-CQA) from green coffee extract are highly bioavailable in humans," Journal of Nutrition, vol. 9, pp. 2309-2315, 2008.

[22] T. Iwashina and S. Matsumoto, "Flavonoid glycosides from the Fern, schizaea (Schizaeaceae) in South Pacific region and their distribution pattern," Food Chemistry, vol. 39, no. 4, pp. 195-201, 2013.

[23] M. N. Clifford, K. L. Johnston, S. Knight, and N. Kuhnert, "Hierarchical scheme for LC-MSn identification of chlorogenic acids," Journal of Agriculture and Food Chemistry, vol. 51, no. 10, pp. 2900-2911, 2003.

[24] R. Jaiswal, H. Muller, A. Muller, M. G. E. Karar, and N. Kuhnert, "Identification and characterization of chlorogenic acids, chlorogenic acid glycosides and flavonoids from Lonicera henryi L (Caprifoliaceae) leaves by LC-MSn," Phytochemistry, vol. 108, pp. 252-263, 2014.

[25] C. Makita, L. Chimuka, P. Steenkamp, E. Cukrowska, and E. Madala, "Comparative analyses of flavonoid content in Moringa oleifera and Moringa ovalifolia with the aid of UHPLC-qTOF-MS fingerprinting," South African Journal of Botany, vol. 105, pp. 116-122, 2016.

[26] H. Kim, E. M. Seo, A. R. Sharma et al., "Regulation of Wnt signaling activity for growth suppression induced by quercetin in 4T1 murine mammary cancer cells," International Journal of Oncology, vol. 43, no. 4, pp. 1319-1325, 2013.

[27] M. I. Yousef, S. A. M. Omar, M. I. El-Guendi, and L. A. Abdelmegid, "Potential protective effects of quercetin and curcumin on paracetamol-induced histological changes, oxidative stress, impaired liver, kidney functions and haematotoxicity in rat," Food and Chemical Toxicology, vol. 48, no. 11, pp. 3246-3261, 2010.

[28] M. Nishimura, T. Ohkawara, Y. Sato et al., "Effectiveness of rutin-rich tartary buckwheat (Fagopyrum tataricum gaertn.) Manten-Kirari in body weight reduction related to its antioxidant properties: a randomised, double-blind and placebo-controlled study," Journal of Functional Foods, vol. 26, pp. 460-469, 2016.

[29] M. Vagiri, S. Conner, D. Stewart et al., "Phenolic compounds in blackcurrant (Ribes nigrum L.) leaves relative to leaf position and harvest date," Food Chem, vol. 172, pp. 135-142, 2010.

[30] V. P. Litvinov, "Chemistry and biological activities of 1, 8naphthyridines," Russian Chemical Reviews, vol. 73, no. 7, pp. 637-670, 2007.

[31] D. K. Sharma, "Pharmacological properties of flavonoids including flavonolignans-integration of petrocrops with drug development from plants," Journal of Scientific and Industrial Research, vol. 65, pp. 477-484, 2006.

[32] Ö. Toku, M. K. Ünal, and Z. Yildirim, "HPLC-UV and GC-MS characterization of the flavonol aglycones quercetin, kaempferol and myricetin in tomato pastes and other tomatobased products," Acta Pharmacologica, vol. 13, pp. 196-207, 2003.

[33] K. Govindaraju, S. Tamilselvan, and V. Kiruthiga, "Biogenic silver nanoparticles by Solanum torvum and their promising antimicrobial activity," Journal of Biopesticides, vol. 3, pp. 394-399, 2010.
[34] Y. Iijima, B. Watanabe, R. Sasaki et al., "Phytochemistry steroidal glycoalkaloid profiling and structures of glycoalkaloids in wild tomato fruit," Phytochemistry, vol. 95, pp. 145-157, 2013.

[35] S. S. Al Sanani, E. A. Eltayeb, Y. T. Kamal, M. S. Khan, and S. Ahmad, "Variations in the cytotoxic glycoalkaloids solamargine and solasonine in different parts of the Solanum incanum plant during its growth and development in Oman," Journal of Taibah University for Science, vol. 10, no. 6, pp. 813-822, 2016.

[36] Y. Chen, F. Sun, H. Han et al., "In-vivo antimalarial activities of glycoalkaloids isolated from Solanaceae plants," Pharmaceutical Biology, vol. 48, no. 9, pp. 1018-1024, 2010.

[37] Dictionary of Natural Products, 2015, http://dnp. chemnetbase.com/dictionary-search.do;jsessionid=A9890 698699B3BE33D7EEAB7ECEBF3B7? $m$ ethod=view\&id=11497129 \&si $=$.

[38] KNApSAcK Metabolite Information Database (Online), "KNApSAcK: a comprehensive species-metabolite relationship database," 2015, http://kanaya.naist.jp/knapsack_jsp/top. html. 


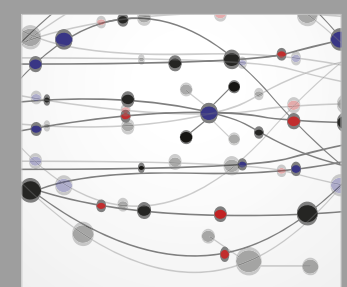

The Scientific World Journal
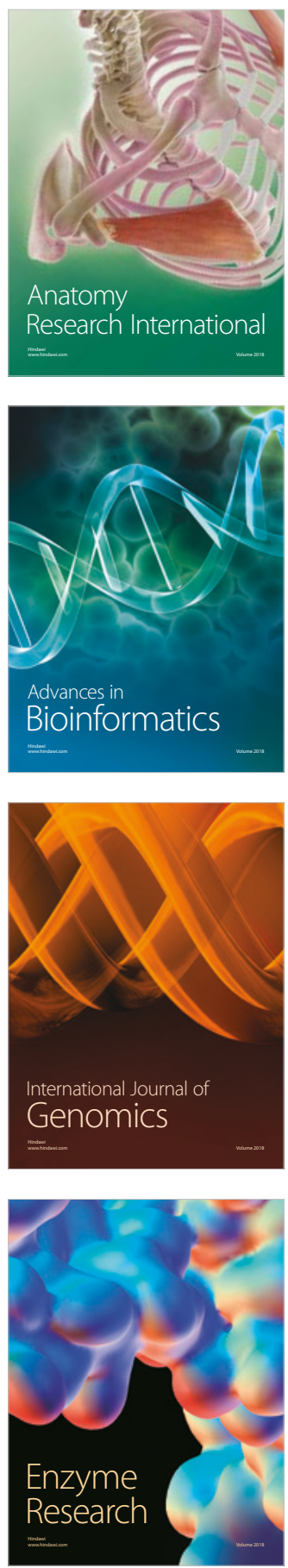
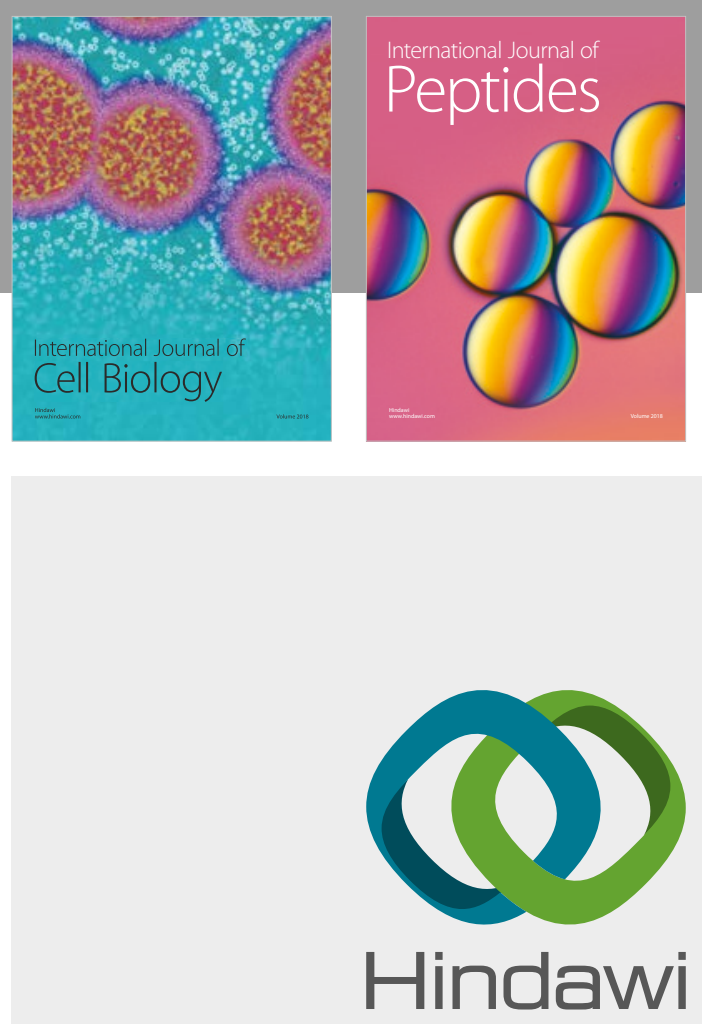

Submit your manuscripts at

www.hindawi.com
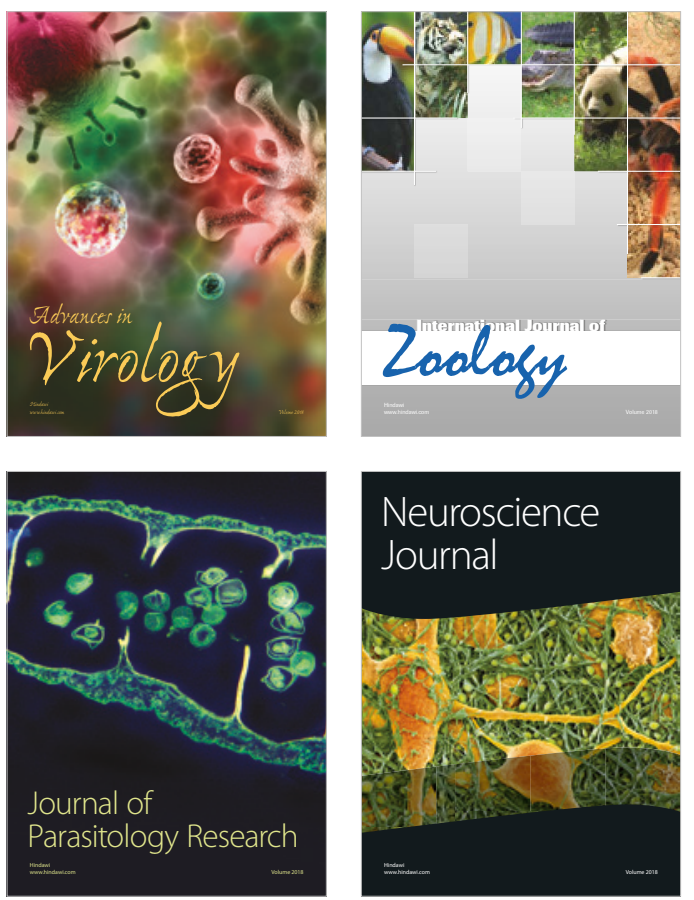
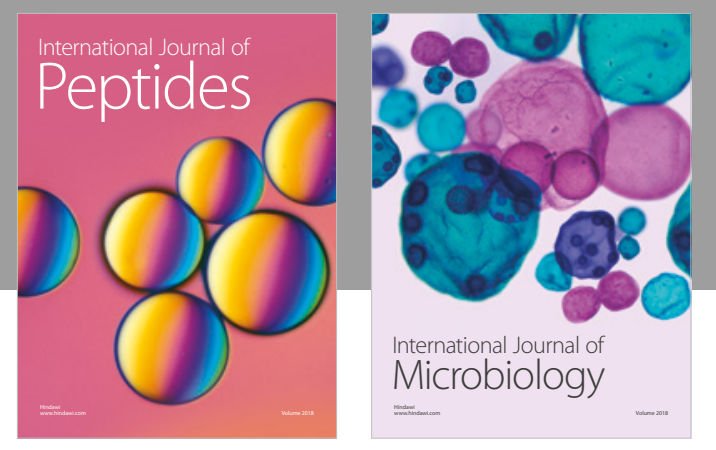

nternational Journal of Microbiology
Journal of

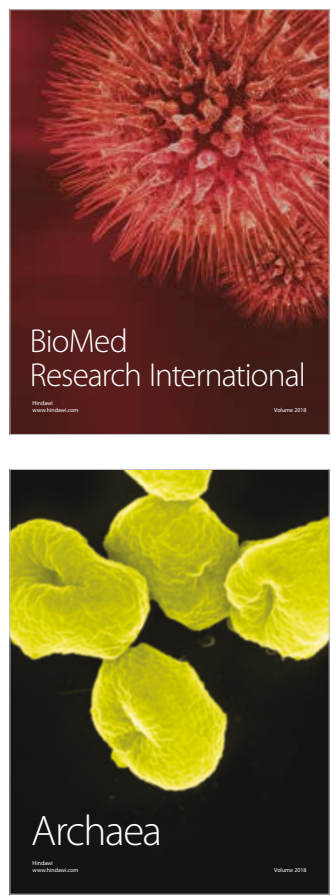